\title{
Inteligencia artificial en bibliotecas: oportunidades como usuarios, y posibles contribuciones
}

"Do you remember that time, way back in the 1990s, when books didn't talk to each other?" (Edward A.

Feigenbaum, 10 de abril 1995).

RICARDO EITO BRUN

GMV AD (Madrid) y Universidad Carlos III de Madrid

\begin{abstract}
Breve descripción de la historia, evolución y progresos del uso de la Inteligencia Artificial en las bibliotecas. Se exponen las posibilidades que existen actualmente para el uso de IA, algunas de las cuales no tienen un reflejo en las estrategias de las bibliotecas. Se analizan las pautas que establece la IFLA Statement on Libraries and Artificial Intelligence que considera la IA como medio de mejorar la recuperación de recursos, automatizar y crear servicios accesibles. Se revisan las iniciativas que se encuadran dentro de las dos grandes líneas de trabajo que recomienda la IFLA: trabajar conjuntamente con los desarrolladores $e$ incluir en las competencias digitales del usuario.
\end{abstract}

Inteligencia artificial, bibliotecas, Feigenbaum, aprendizaje automático, reconocimiento de voz, makerspaces, declaraciones IFLA.

En los últimos años se observa una interesante discusión, acompañada de avances reales, en torno a las posibilidades de la inteligencia artificial (en adelante IA) en distintas áreas. Con una creciente aparición en los medios, esta tecnología nos presenta desarrollos sorprendentes y programas realmente innovadores. El Diccionario Español de Ingeniería recoge dos acepciones para la IA: "conjunto de técnicas que tienen por objeto dotar a un sistema informático de la capacidad de simular algunas características que se suponen propias de la inteligencia humana" y “área de la informática que ... genera soluciones a problemas mediante paradigmas generales de representación de conocimiento...". Junto a otras tecnologías disruptivas como blockchain, la robótica o la internet de las cosas, la IA es uno de los elementos llamados a hacer posible la "cuarta revolución industrial" capaz de introducir mejoras radicales en la forma en la que 
desarrollamos nuestras actividades, y en cómo prestamos servicios a la comunidad de usuarios. ¿Son aplicables estas promesas y estos beneficios en nuestro ámbito profesional?

En 1994, Edward Feigenbaum - uno de los padres de la IA -, presentó un escenario hipotético en el que se situaba en el futuro (concretamente en el año 2020), para formular esta pregunta: "¿recuerdas esos tiempos, en los años noventa, cuando los libros no hablaban unos con otros?" En su reflexión, Feigenbaum establecía una distinción entre los “libros pasivos", que no hacían nada para el lector - que se veía obligado a usar su inteligencia para extraer información y datos útiles -, y los "libros activos", capaces de comprender el contexto de nuestras actividades y ofrecernos ayuda y conocimiento... Obviamente, la visión de Feigenbaum queda aún lejana, pero son innegables los progresos alcanzados en la aplicación y uso de la IA en bibliotecas y centros de documentación. El reconocimiento otorgado a la Dirección de Documentación de Atresmedia con el XV Premio Nacional SEDIC a la Calidad e Innovación "Carmen Rodríguez Guerrero" es una excelente muestra de ello.

Si tuviésemos que describir la historia y evolución del uso de la IA en nuestra área, podríamos hablar de un progreso continuado, aunque en la percepción de los profesionales exista el riesgo de considerarlo como un tema de interés reciente, consecuencia de una moda que, como otras, podría verse relegada a un segundo plano más adelante. Frente a esto, hay que señalar que - a pesar de lo novedoso que hoy nos resulta y de la popularidad alcanzada en los últimos años -, las propuestas para aplicar la IA en sus distintas manifestaciones (sistemas expertos basados en reglas, sistemas de recomendación, etc.) están presentes en nuestra disciplina desde hace unos cincuenta años. 


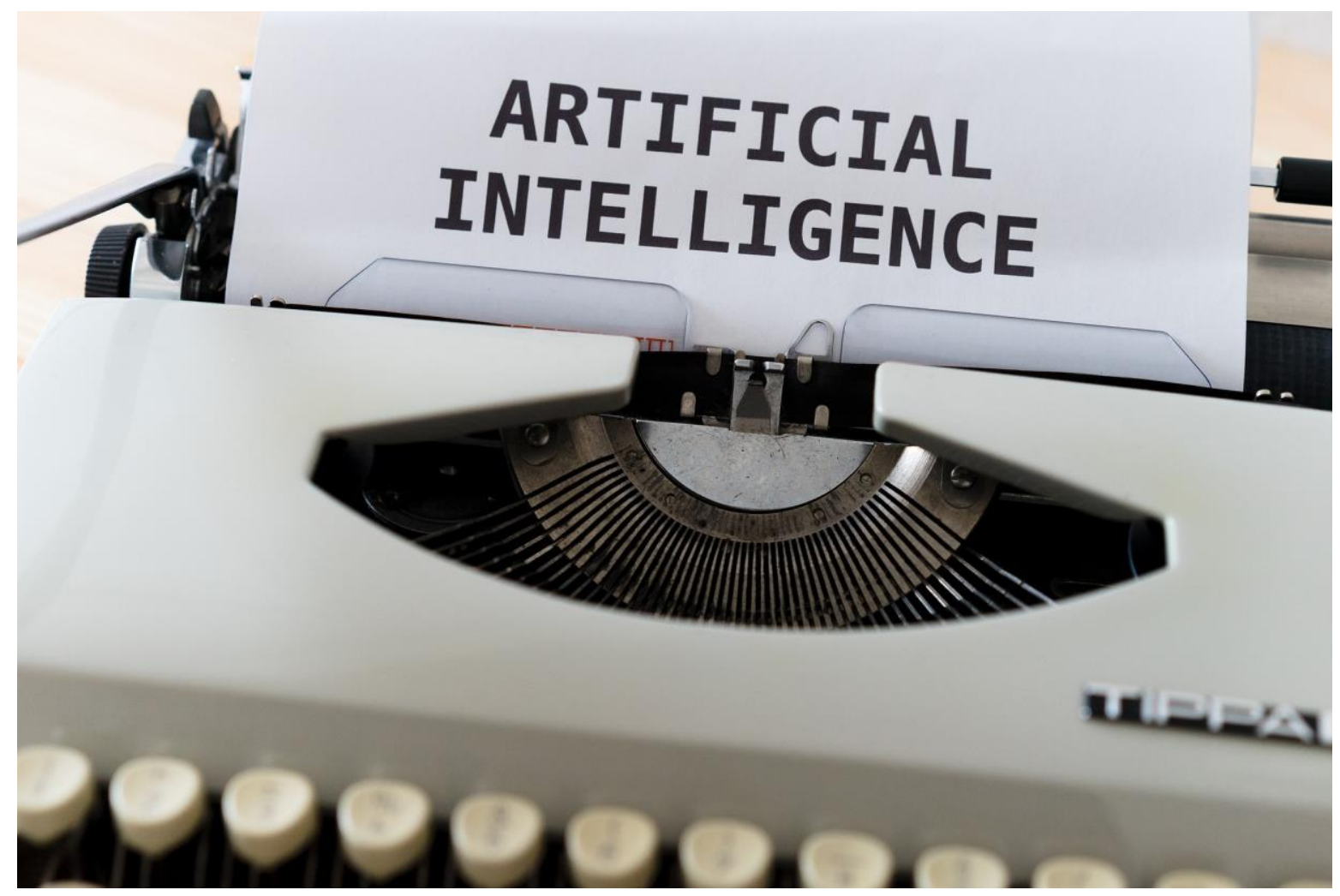

Una búsqueda en la base de datos LISA (Library Information Science Abstracts) nos presenta más de diez mil resultados; los primeros trabajos de nuestra área en los que se menciona "inteligencia artificial” se publicaron en 1976 y 1977, entre ellos: Artificial intelligence in information retrieval systems y Man-machine communication: the artificial intelligence approach.

En la década de los ochenta encontramos más iniciativas, la mayoría dedicadas al estudio de sistemas expertos capaces de actuar como intermediarios entre usuarios, bases de datos y catálogos (Meadow, 1982; Pollitt, 1984; Borko, 1985). También fueron objeto de discusión los sistemas question-answering capaces de extraer datos a partir de textos y responder a preguntas específicas de los usuarios.

También durante la década de los noventa se publicaron numerosas contribuciones y monografías: Expert Systems in Libraries (Aluri y Riggs, 1990), The Application of Expert Systems in Libraries and Information Centres (Morris, 1992) o Knowledge-Based Systems for General Reference Work: Applications, Problems, and Progress (Richardson, 1995), en las que se presentaban aproximaciones para mejorar los servicios bibliotecarios: sistemas de recomendación para servicios de referencia, ampliación de ecuaciones de búsqueda con términos derivados a partir de la relevancia que los usuarios otorgaban a resultados intermedios 
(relevance feedback), elaboración automática de resúmenes, indización, clasificación e incluso catalogación, sin intervención humana, etc.

En la literatura en castellano también encontramos contribuciones, como el artículo Una visión hacia el futuro: los sistemas expertos, (Montoro, 1990), o la revisión de Lopez Guallamón (2004) que incluía un interesante epígrafe titulado “catalogación sin catalogador”. Otra actividad que se debe destacar es la investigación desarrollada por Carmen Díez Carrera y Carlos González Ruiz, publicada en forma de monografía por la editorial TREA: Investigación y gestión de proyectos en biblioteconomía: el caso de la catalogación automática (DIC).

En numerosos casos, la IA se asoció con tecnologías para el procesamiento textual que no siempre han sido etiquetadas con ese término: clasificación automática, clustering, explotación de vocabularios controlados, etc.

En la actualidad, si bien se siguen presentando iniciativas orientadas a la mediación entre usuarios y sistemas de recuperación, la aplicación de la IA en bibliotecas presenta nuevas posibilidades. Esto se debe a que el alcance de la IA ha pasado a incluir un conjunto más amplio de tecnologías; el término ya no se limita a sistemas de carácter determinista basados en reglas heurísticas, sino que incorpora el reconocimiento de patrones en imágenes, la visión artificial, el reconocimiento de voz, y el aprendizaje automático (Machine Learning) en general. Esta redefinición de su alcance hace que cualquiera de nosotros seamos usuarios habituales de la IA: cuando usamos el filtrado de spam en nuestro correo electrónico, las recomendaciones de las redes sociales o los agentes conversacionales (chatbots) que han incorporado numerosos sitios web. Un segundo factor que contribuye a los nuevos usos de la IA responde al hecho de que se han identificado y se muestra una mayor sensibilidad a otro tipo de necesidades, aparte de las derivadas de la complejidad de la recuperación de información.

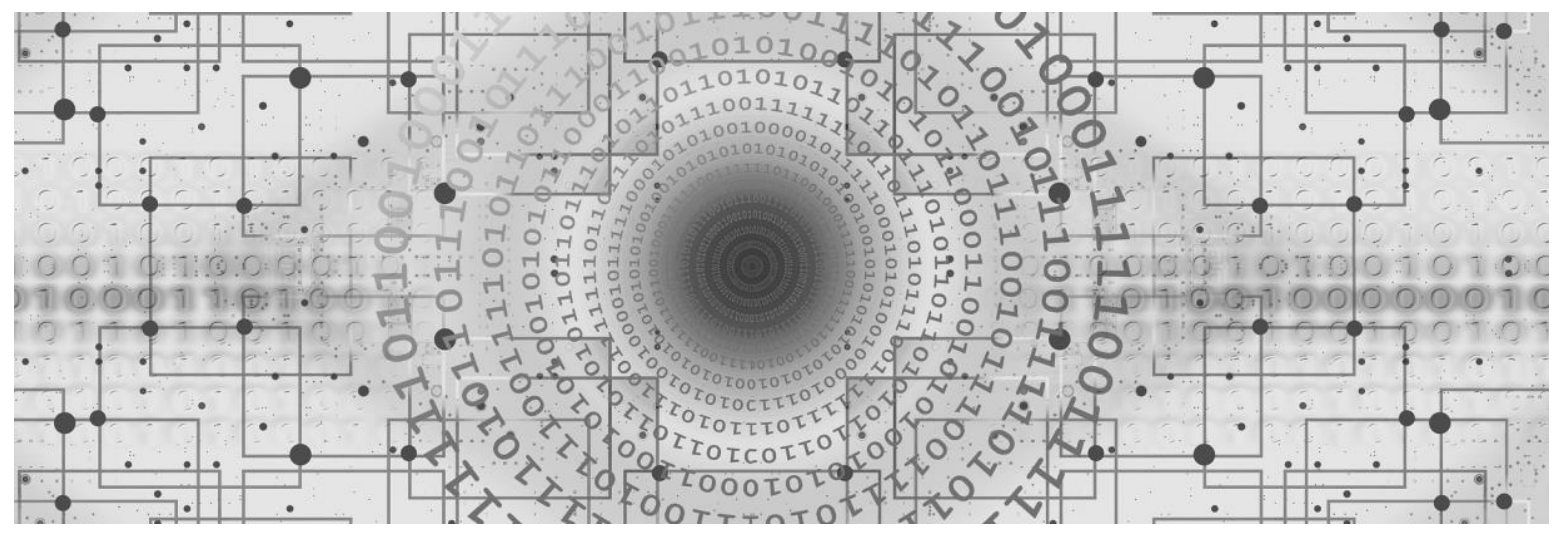




\section{¿Qué ha sucedido recientemente?}

Wheatley, A.; Hervieux, S. y Lawlor (2019) analizaron la presencia de la IA en las políticas, estrategias y actividades de distintas bibliotecas universitarias norteamericanas y canadienses. Aunque identificaron iniciativas relacionadas con el uso de chatbots y ofertas puntuales de seminarios sobre el tema, señalaron que la IA no se mencionaba en ninguno de sus planes estratégicos y que únicamente un centro había elaborado una guía sobre esta temática. Los autores concluyeron, con cierta rotundidad, que "el estado actual de la IA en bibliotecas universitarias ha demostrado ser prácticamente inexistente". En ese mismo año, el informe Artificial Intelligence and Machine Learning in Libraries (Griffey, 2019) - centraba su impacto en las áreas tradicionales: mediación en la recuperación y alternativa para la catalogación, indización y referencia. La mediación entre el usuario y las colecciones de documentos no ha dejado de estar presente, y se pueden citar como ejemplos las aplicaciones software Yewno Discover o iris.ai.

La revisión - no exhaustiva - de las contribuciones publicadas en el último año en las que se hace referencia explícita a la IA en bibliotecas, nos permite identificar otras líneas de trabajo. El primer documento interesante es la declaración publicada por IFLA el diecisiete de septiembre de 2020 (IFLA Statement on Libraries and Artificial Intelligence), en la que se invita a considerar la IA como un medio para mejorar la recuperación de recursos, automatizar y crear servicios accesibles. Para ello se recomienda el trabajo conjunto con desarrolladores de IA, e incluirla entre las competencias digitales de los usuarios. En su anexo III, se mencionan cinco iniciativas IA: la automatización de la clasificación bibliográfica por parte de la biblioteca nacional de Noruega, el Al Lab de la universidad de Rhode Island, el sistema de discovery Hamlet del MIT para la recuperación de tesis, la aplicación móvil de la Helsinki Central Library Oodi que sugiere lecturas a los usuarios y el Al Studio de la biblioteca universitaria de Stanford. Este último propuso explorar - mediante distintos proyectos - la capacidad de clasificar contenidos mediante aprendizaje automático basado en patrones.

El resto de las iniciativas se pueden encuadrar en dos grandes líneas de trabajo. La primera se centra en la interacción del usuario con las bibliotecas mediante reconocimiento de voz, agentes conversacionales y dispositivos wearables. Los ejemplos incluyen el lector OrCam My Eye la de la St. Clair Country's Library (Michigan), que facilita la lectura a personas con discapacidad visual u otras dificultades (Howard, 2020). El uso del reconocimiento de voz para consultar el 
catálogo, renovar préstamos, obtener información general o incluso localizar documentos en el depósito, se ha incorporado a distintas aplicaciones informáticas (Rivero, 2020). También las plataformas de recursos electrónicos como EBSCO o ExLibris han incorporado esta capacidad en algunos productos. Otros ejemplos relacionados con la interacción incluyen referencias a los agentes conversacionales, como el descrito por Matt LaBrake (2019) - director de la Online Library Berkeley College - quien señaló acertadamente que el éxito de su iniciativa se debió, en gran parte, a la completitud y extensión de la base de conocimiento creada por el personal de la biblioteca para atender su servicio de referencia. Este aspecto merece destacarse, ya que la utilización exitosa de algunas de estas tecnologías exige un trabajo sistemático previo y estructurar e integrar la información para dar respuesta a las peticiones que planteen los usuarios.

La segunda línea de trabajo busca incorporar la IA en las competencias digitales de los usuarios, y promover su aprendizaje por parte de la comunidad. Se materializa en la puesta en marcha de Makerspaces para desarrollar y promover proyectos IA. Encontramos referencias como la de la Frisco Public Library (Texas) - que facilita kits Google AIY (Artificial Intelligence Yourself) para reconocimiento de voz y video - (Finley, 2019), o el Al Lab de la universidad de Rhode Island citado en la declaración de IFLA (Bohyun, 2020).

A parte de estas líneas, encontramos otros usos de la IA en tareas como el control del inventario puesto en marcha por la biblioteca japonesa de Funabashi, basado en drones, y citado en el número de junio de 2020 de American Libraries.

Para concluir, se debe reflexionar sobre otra actividad relacionada con la IA que afecta directamente a nuestro sector profesional, no como usuarios de la IA, sino como facilitadores de esta. Muchas aplicaciones IA se basan en la disponibilidad de datos, en su calidad y accesibilidad; esto se alinea con la necesidad de gestionar y preservar esos conjuntos de datos de forma que se garantice su uso, comprensión y reutilización. Las nuevas responsabilidades que la comunidad profesional viene adoptando de forma progresiva en la custodia y tratamiento de datos, unidas a las políticas de acceso abierto y a las estrategias de preservación, están llamadas a contribuir y a lograr un uso cada vez más inteligente de las tecnologías. 


\section{Referencias}

Aluri, R. y Riggs, D. (1990). Expert Systems in Libraries. Ablex, 352 p.

Augier, M-S., \& Vendelø, M. T. (2002). An interview with Edward A. Feigenbaum. Disponible en: https://research.cbs.dk/en/publications/an-interview-with-edward-a-feigenbaum

Bohyun K. (2020). Moving Forward with Digital Disruption: What Big Data, loT, Synthetic Biology, Al, Blockchain, and Platform Businesses Mean to Libraries. Library Technology Reports. 56(2): 1-32.

Borko, H. (1985). Artificial intelligence and expert systems research and their possible impact on information science. Education for Information. 3(2):103-114.

Díez Carrera, C. (2006). Investigación y gestión de proyectos en biblioteconomía el caso de la catalogación automática (DIC). Trea, 80 p.

Finley, T. (2019). The Democratization of Artificial Intelligence: One Library's Approach. Information Technology \& Libraries. 38(1): 8-13.

Griffey, J. (ed.) (2019). Artificial Intelligence and Machine Learning in Libraries. ALA TechSource, 32 p.

Howard, M. (2020). Harnessing the Power of OrCam. Information Technology \& Libraries. 39(3):13.

IFLA (2020). IFLA Statement on Libraries and Artificial Intelligence. Disponible en: https://www.ifla.org/publications/node/93397

Labrake, M. (2019). Getting your FAQs straight: how to make your knowledgebase power virtual reference. Computers in Libraries. 39(8): 14-19.

López Guillamón, I. (2004). Una nueva catalogación: objetivos, principios, características y control de autoridad. Revista Española de Documentación Científica, 27(2): 192-211.

Meadow, Ch. T.; Hewett, T. y Aversa, E. (1982). A Computer Intermediary for Interactive Database Searching. Journal of the American Society for Information Science. 33(6): 357-364.

Montoro, I. (1990). Una Visión Hacia el Futuro: Ios Sistemas Expertos. Cuadernos de la ADAB. 34: 51-62.

Morris, A. (1992). The Application of Expert Systems in Libraries and Information Centres. K. G. Saur, $241 \mathrm{p}$. 
Mylopoulos, J. y Perrault, C. R. (1977). Man-machine communications: the artificial intelligence approach. Canadian Journal of Information Science. 2(1): 120-128.

Pollitt, A S. (1984). A 'front end' system. An Expert System as an online search intermediary. Aslib Proceedings. 36(5): 229-234.

Richardson, J. (1995). Knowledge-Based Systems for General Reference Work: Applications, Problems, and Progress. Academic Press, 355 p.

Rivero, E. (2020). Library and Educational Use Cases. Library Technology Reports. 56(4):14-24.

Smith, L. C. Artificial intelligence in information retrieval systems. (1976). Information Processing \& Management. 12(3):189-222.

Wheatley, A.; Hervieux, S. y Lawlor, B. (2019). Artificial intelligence in academic libraries: An environmental scan. Information Services \& Use. 39(4): 347-356.

\section{Sobre el autor}

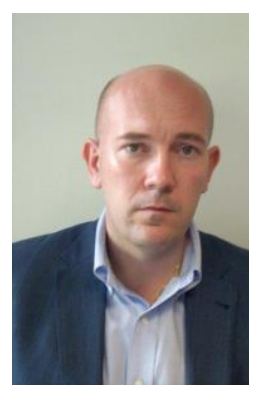

\section{RICARDO EITO BRUN}

Desempeña su actividad profesional en GMV AD (Madrid), en el área de calidad y proyectos de Sistemas de Información. Anteriormente formó parte del área de I+D de Meta4 Software (Madrid) y de Adecco WWIT. Es profesor asociado en la Universidad Carlos III de Madrid, Departamento de Biblioteconomía y Documentación desde 1999.

Doctor en Sistemas de Información y Documentación por la Universidad de Zaragoza. Doctor en Ciencias de la Computación por la Universidad de Alcalá de Henares. MSc Software Engineering Technische Universitaet Kaiserslautern - IESE (Institute for Experimental Software Engineering) MsC Ciencia y Tecnología Informática por la Universidad Carlos III de Madrid. Licenciado en Documentación por la Universidad de Granada.

- www.linkedin.com/in/ricardo-eito-brun

- https://portal.uczm.es/portal/page/portal/biblioteconomia_documen tacion/profesores/reito 